\title{
Application of Instantaneous Rotational Speed to Detect Gearbox Faults Based on Double Encoders
}

Lin Liang ${ }^{1,2^{*}}$, Fei Liu' ${ }^{1}$ Xiangwei Kong ${ }^{1}$, Maolin $\mathrm{Li}^{3}$ and Guanghua $\mathrm{Xu} \mathrm{u}^{1,4}$

\begin{abstract}
Considerable studies have been carried out on fault diagnosis of gears, with most of them concentrated on conventional vibration analysis. However, besides the complexity of gear dynamics, the diagnosis results in terms of vibration signal are easily misjudged owing to the interference of sensor position or other components. In this paper, an alternative gearbox fault detection method based on the instantaneous rotational speed is proposed because of its advantages over vibration analysis. Depending on the timer/counter-based method for the pulse signal of the optical encoder, the varying rotational speed can be obtained effectively. Owing to the coupling and meshing of gears in transmission, the excitations are the same for the instantaneous rotational speed of the input and output shafts. Thus, the differential signal of instantaneous rotational speeds can be adopted to eliminate the effect of the interference excitations and extract the associated feature of the localized fault effectively. With the experiments on multistage gearbox test system, the differential signal of instantaneous speeds is compared with other signals. It is proved that localized faults in the gearbox generate small angular speed fluctuations, which are measurable with an optical encoder. Using the differential signal of instantaneous speeds, the fault characteristics are extracted in the spectrum where the deterministic frequency component and its harmonics corresponding to crack fault characteristics are displayed clearly.
\end{abstract}

Keywords: Instantaneous rotational speed, Optical encoder, Localized fault, Multistage gearbox

\section{Introduction}

As the transmission component widely used in various machines, the gearbox plays a critical role in mechanical systems, and thus, gearbox fault diagnosis based on vibration signals attracts considerable research interests [1]. In addition to deterministic approaches such as statistical features $[2,3]$ and time-frequency analysis $[4,5]$, many analysis tools have been used, including decision tree [6], frequency demodulation [7,8], and stochastic resonance $[9,10]$. However, vibration analysis still has inherent limitations in the diagnosis of complex gear system, in which the vibration signal is easily contaminated by a large amount of interference, and the strength

\footnotetext{
*Correspondence: lianglin@xjtu.edu.cn

${ }^{1}$ School of Mechanical Engineering, Xi'an Jiaotong University, Xi'an 710049, China

Full list of author information is available at the end of the article
}

of the signal depends on the position of the vibration transducer.

As the torsional vibration signal has a simpler spectral structure than the transverse vibration signal [11] and has the advantage of a short transmission path and high signal-to-noise ratio, it is also applied to the fault diagnosis of gears in wind turbines $[12,13]$ and traction systems [14]. Recently, the instantaneous angular speed (IAS) has revealed a high sensitivity to change in mechanical systems [15]. Because the defects in rotating machines have a direct impact on the angular speed, the IAS signal usually contains a considerable amount of information on fault components [16]. The different IAS-based techniques have been developed for bearings [17], induction motors [18], and gearboxes. For the gear transmission of numerical control machines, Zhou et al. [19] utilized the instantaneous speed obtained using a servo motor encoder 
to monitor the state of the parts, and adopted the instantaneous speed estimated by the digital differentiator to identify the vibration source [20]. Additionally, Stander et al. [21] used a simplified mathematical model of a gear system to illustrate monitoring of the instantaneous angular speed. Roy et al. [22] applied spectrum to the synchronously averaged IAS signal under different working conditions.

Recently, Li et al. [23] developed a new approach to extract the features of a gearbox based on IAS signal. The idea behind using IAS signal is that the presence of a defect changes the dynamics of the gearbox and this definitively alters the rotational speeds of the shafts. Besides the IAS signals measured from the input shaft of the gearbox, Sankar et al. [24] also analyzed the varying speed of the output shaft for detection of faults in a multistage automobile gearbox. However, considering the gear stage, the unavoidable interference in the gear chain can contribute to the signal of instantaneous angular speed, which increases the difficulty of feature extraction.

It is verified that a localized gear fault can result in a periodic fall in the mesh stiffness at the rotational frequency of the faulty gear shaft, resulting in a change in the instantaneous rotational speed; this indicates that it can be used to detect the presence of a gear defect. Consequently, as an angular sensor, the optical encoder offers high resolution and accuracy with the mechanism of data acquisition, and the timer/ counter-based method is used to obtain the associated instantaneous rotational speed directly. Thus, for the transmission system, the instantaneous speed of the input and output shafts can be obtained by optical encoders synchronously. Moreover, to reduce the effects of external interference and improve the ability of fault detection for gear transmission systems, the differential signal of the instantaneous speeds of the input and the output shafts is utilized to extract the gear fault feature. Compared with the signal of a single encoder, instantaneous transmission ratio, and vibration signal, the proposed feature extraction method based on the differential signal of double encoders is feasible and effective.

This paper is organized as follows: Section 2 provides the fundamental knowledge of the rotating speed fluctuation of gear defects. In Section 3, the principle of the measurement of instantaneous speed is introduced. Section 4 proposes the feature extraction scheme based on the differential signal of instantaneous speeds in detail, and the gear defect experiment is presented to evaluate the proposed method in Section 5. Finally, conclusions are drawn in Section 6.

\section{Rotational Speed Fluctuation of Gear Defect}

For the gear transmission, the input shaft transmits torque to the output shaft with meshing gears $[25,26]$, and the model of single axis transmission system can be given by

$$
\boldsymbol{J} \ddot{\Omega}+\boldsymbol{C} \dot{\Omega}+k \Omega=M,
$$

where $\boldsymbol{J}$ and $\boldsymbol{C}$ are the rotational inertia and damping matrix, respectively; $\Omega$ and $M$ are the angular displacement and load, respectively; and $k$ is the mesh stiffness. For a gear mesh period $T_{m}$ and a given contact ratio $(1<\varepsilon<2)$, the pinion and wheel are first contacted by one pair of teeth during $(\varepsilon-1) T_{m}$; then, both are contacted by two pairs of teeth during $(2-\varepsilon) T_{m}$. Thus, gear mesh stiffness is periodic with the period $T_{m}$, which can be approximated as

$$
k(t)=\left\{\begin{array}{l}
k_{\max }, \text { if } n T_{m} \leq t \leq(n+\varepsilon-1) T_{m}, \\
k_{\min }, \text { if }(n+\varepsilon-1) T_{m} \leq t \leq(n+1) T_{m},
\end{array}\right.
$$

where $n$ is an integer representing the $n$th gear mesh period [27]. It is well known that a broken tooth can lead to a loss of contact, and consequently a periodic loss in tooth stiffness. The tooth crack also leads to a periodic decline in the gear mesh stiffness with the rotational frequency of the faulty gear, which results in periodical perturbation of speed with rotation. It follows that the external excitations are mainly attributed from the abnormal change in mesh stiffness when the load and input speed are constant. Therefore, the rotational speed theoretically includes not only the mesh frequencies, but also the low frequency component corresponding to the rotation of the faulty gear.

\section{Measurement of Instantaneous Speed Based on the Encoder}

\subsection{Pulse Sequence of Shaft Angular Displacement}

For multi-stage gear transmission systems, optical encoders with same resolution are mounted on the input and output shafts of the gearbox respectively. It is assumed that the teeth of the grating disc are uniformly distributed around the circumference. Then, without considering the effects of noise, the output signal of the shaft angular displacement from the optical encoder exhibits a uniformly periodic pulse $f(t)$ when the angular speed of the rotary shaft is constant. Thus, the signal of the shaft encoder can be represented by [28]

$$
f(t)=\sum c_{n} \cos (n r \omega t+\theta)
$$

where $\omega$ and $\theta$ are the angular speed of the rotary shaft and initial phase of the signal, respectively; $r$ is the 


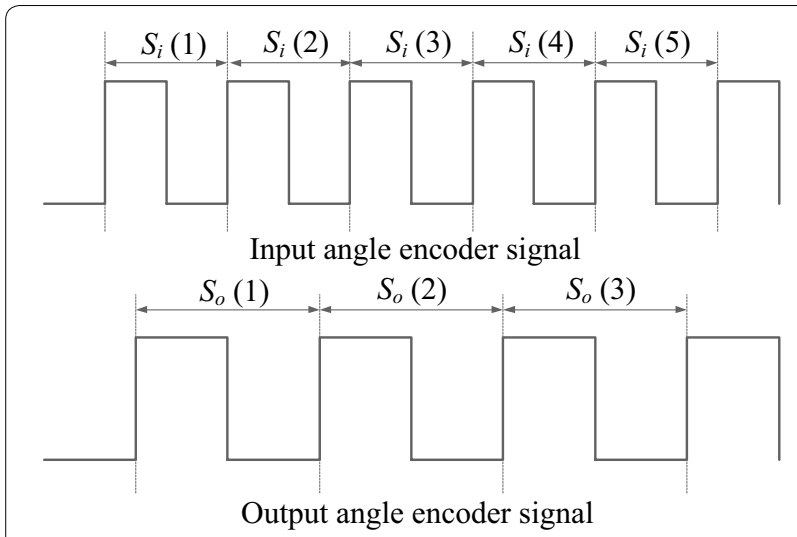

Figure 1 Pulse sequence of shaft angular displacement

resolution of the encoder; and $c_{n}$ is the amplitude of the $n$th harmonic component. Note that the pulse period of the sequence of shaft angular displacement corresponds to the rotational speed. Figure 1 shows the pulse period $S$ of the angular displacement of the input and output shafts, where $S_{i}=\left\{S_{i}(1), S_{i}(2), S_{i}(3), \ldots\right\}$, and $S_{o}=\left\{S_{o}(1), S_{o}(2), S_{o}(3), \ldots\right\}$. It can be seen that each $S$ is the elapsed time (ET) between two successive pulses in the prescribed duration. Generally, when a tooth mesh has a faulty tooth, the mesh stiffness suddenly changes, causing speed fluctuation, and this is reflected by the change in corresponding elapsed time. Thus, it enables the sequence $S$ of successive pulse periods to contain the dynamic responses of the fault components.

\subsection{Measurement of Instantaneous Speed}

Based on the mechanism of data acquisition, the timer/ counter-based method treats the output signal from the optical encoder as a pulse sequence, and the pulse sequence can be used to start and stop the timer/counter. Thus, the instantaneous speed is estimated by measuring the ET for the corresponding unit of angular displacement. In this study, the ET of a speed pulse is obtained by using a high-frequency clock, as shown in Figure 2 . The data acquisition system consists of a high-frequency counter device, which is commonly found on traditional data acquisition boards. The latch circuit latches the time at which the encoder pulses reach a rising edge, and the ET of a single period of encoder signal can be measured. Thus, the ET sequences of the pulse period for the input and output shafts can be extracted synchronously.

As only the revolution speed fluctuations are of interest in this study, all signal processing and analysis is conducted within the time duration of successive encoder gratings. It is known that the frequency of pulse sequence is equal to the reciprocal of ET between two successive

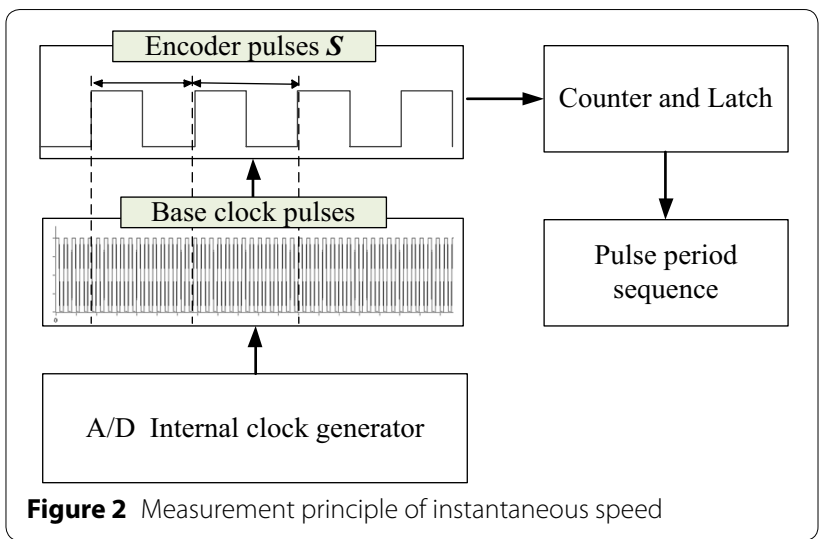

pulses. According to the pulses per revolution of the encoder, the instantaneous revolution speed $V$ of the rotary shaft can be calculated as

$$
V(n)=\left(\frac{1}{S^{\prime}(n)}\right) / r, n=0,1,2, \ldots, N-1,
$$

where $N$ is the number of data points; the resolution $r$ of the encoder is the number of encoder gratings; and the elapsed time of each $S^{\prime}(n)$ is equal to the average of adjacent points $S^{\prime}(n)=(S(n)+S(n+1)) / 2$. Note that the instantaneous speed $V$ is described in the unit of $\mathrm{Hz}$. Obviously, the acquisition process is equivalent to the time estimation between successive rising edges of the encoder signal. It can be seen that the acquisition occurs at a sampling frequency directly linked to the shaft rotational speed and obviously to the resolution of the encoder [29].

\section{Feature Extraction Scheme Based on the Differential Signal of Instantaneous Speeds}

As a component in the transmission device, besides the gear localized defect, some unavoidable manufacturing or installation errors tend to affect the measured instantaneous speed signal, which makes the detection of gear defects difficult. However, considering the common excitation source in the gear chain, the differential signal of instantaneous speeds of the input and output shaft can effectively reduce the effect of these excitation sources, and improve the accuracy of the fault identification. The feature extraction scheme based on the double encoder is shown in Figure 3.

1) The pulse period sequences $S_{i}(n)$ and $S_{o}(n)$ of the input and output shafts, respectively, are measured synchronously; then, the respective instantaneous revolution speeds $V_{i}(n)$ and $V_{o}(n)$ are calculated using Eq. (4). 


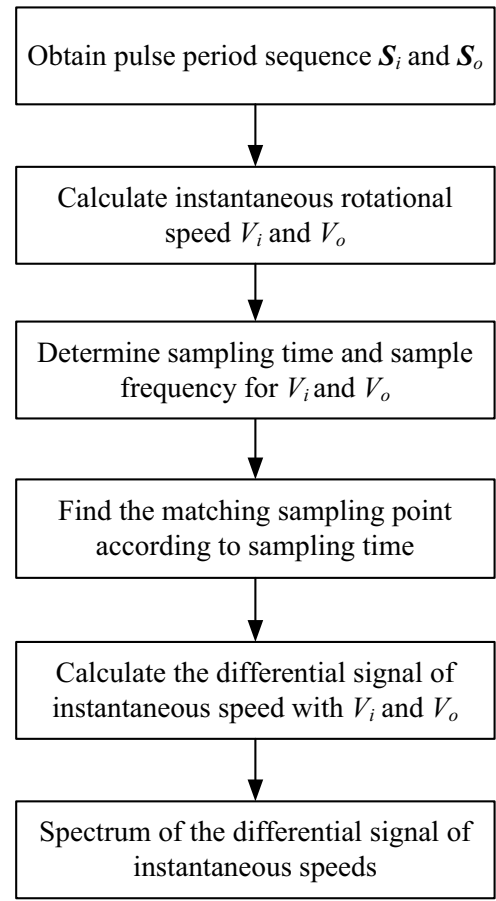

Figure 3 Feature extraction scheme

2) According to the acquisition of the instantaneous speed, the corresponding sample time of each $V(n)$ is equal to the accumulation of corresponding ET of the pulse period. Then, the sample time $T(n)$ of the instantaneous speed can be described as follows:

$T(n)=\sum_{i=0}^{n} S(i), n=0,1,2, \ldots, N-1$.

For different instantaneous speeds $V_{i}(n)$ and $V_{o}(n)$, the corresponding time signals $T_{i}(n)$ and $T_{0}(n)$, respectively, can be calculated using Eq. (5).

3) For simplicity of analysis, the spectrum is obtained by considering an average sampling frequency, which is estimated from the average speed of rotation because the gearbox operates at a constant speed. Based on the mechanism of data acquisition, the timer/counter-based method can sample $r$ points in one revolution of the gear shaft.

Simultaneously, the average rotational speed $\bar{V}$ in revolutions per second is set to $\frac{1}{N} \sum_{i=0}^{N-1} V(i)$.

Then, the associated sampling frequencies of the input and output shafts, respectively, are calculated by multiplying the average rotational speed by the pulse per revolution of the encoder:

$f_{s}=\bar{V} * r$.

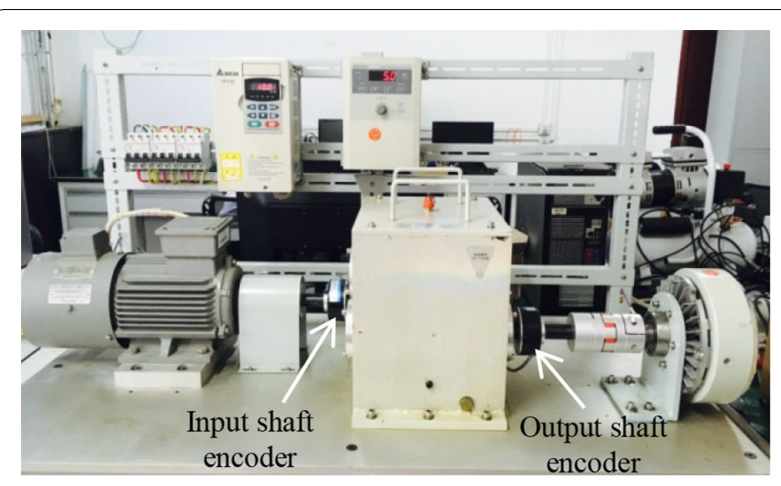

Figure 4 Two-stage spur gearbox test system

4) According to the time signals $T_{i}(n)$ and $T_{o}(n)$ of the instantaneous speeds, pairs of sampling points from the signals of the input and output shafts are determined.

4.1) It is assumed that the sampling frequency for the input shaft is higher; then, the instantaneous speed $V_{i}(n)$ of the input shaft can be set as the basis.

4.2) Time signals $T_{i}(n)$ and $T_{o}(n)$ are used to find the sampling point of the signal $V_{o}(n)$ whose sampling time is closest to that of the signal $V_{i}(n)$. Specifically, the matching sampling points from the output shaft signal, which has the minimum sampling time difference between instantaneous speed signals, are selected.

5) With the pairs of sampling points of the input and output shafts, the differential signal of the instantaneous rotational speed is determined by

$V_{d}\left(n_{i}\right)=g \cdot V_{o}\left(n_{o}\right)-V_{i}\left(n_{i}\right)$,

where $g$ is the transmission ratio; and $V_{i}\left(n_{i}\right)$ and $V_{o}\left(n_{o}\right)$ are the $n$th instantaneous speed points of the input and output shafts, respectively. Thus, the

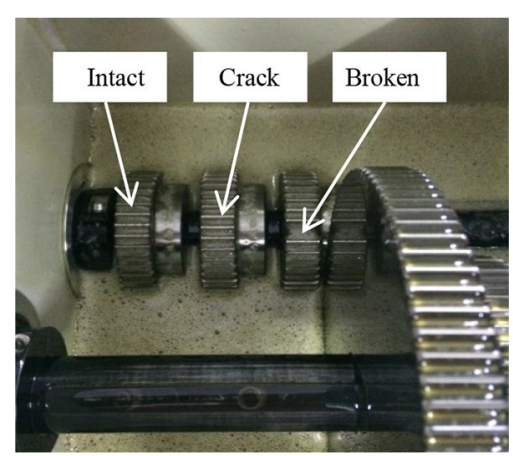

Figure 5 Intact and localized defect gears 


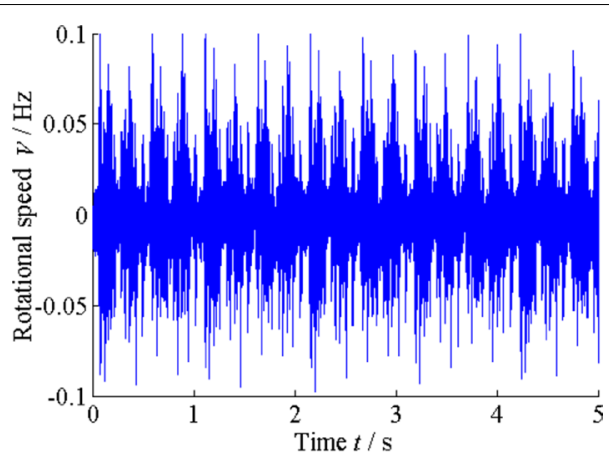

a Waveform of the intact gear

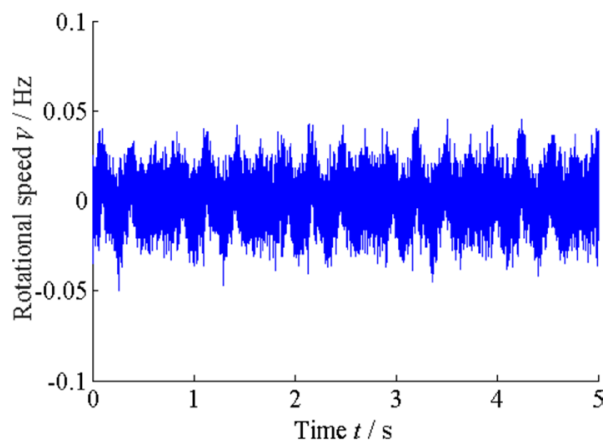

b Waveform of the crack defect

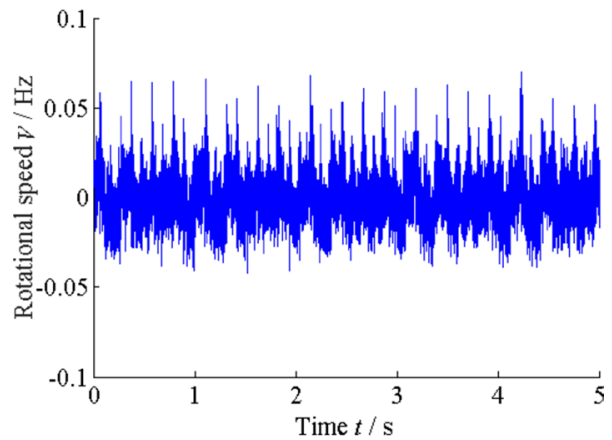

c Waveform of the breakage defect

Figure 6 Differential signals at a speed of $10 \mathrm{~Hz}$

interference can be reduced by the differential signal of instantaneous speeds.

6) The spectrum of differential signal is calculated using the associated sampling frequency $f s$, and the gear defect feature can be easily identified according to the rotational frequency of the rotor shafts.

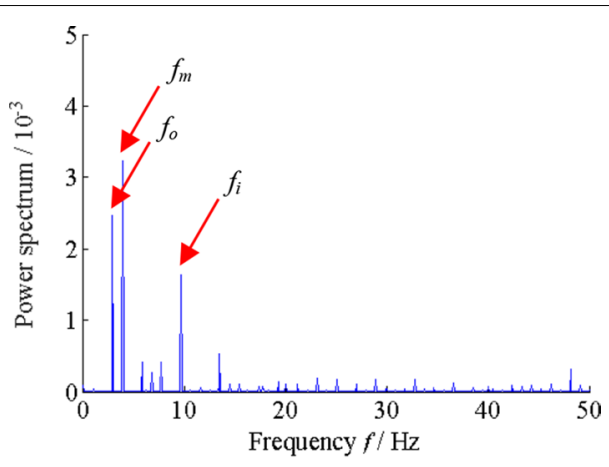

a Spectrum of the intact gear

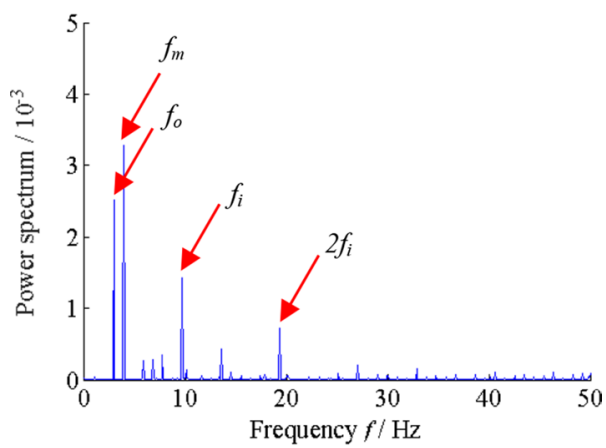

b Spectrum of the crack defect

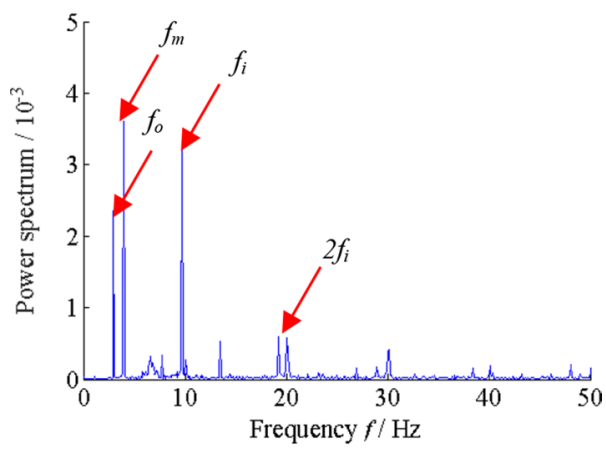

c Spectrum of the breakage defect

Figure 7 Spectra of differential signals at a speed of $10 \mathrm{~Hz}$

\section{Application for Detection of Gearbox Defects 5.1 Experimental Setup}

The main components of the spur gearbox test system are shown in Figure 4. The system primarily includes a twostage spur gearbox that can be used to simulate different localized defects, a 3-hp motor to drive the gearbox, and a magnetic brake to load the torque. The motor rotating speed is controlled by a speed controller. The load is provided by the magnetic brake and can be adjusted by the brake controller. The transmission ratio of the gearbox is 3.3. Two encoders with a resolution of 1024 pulse per 


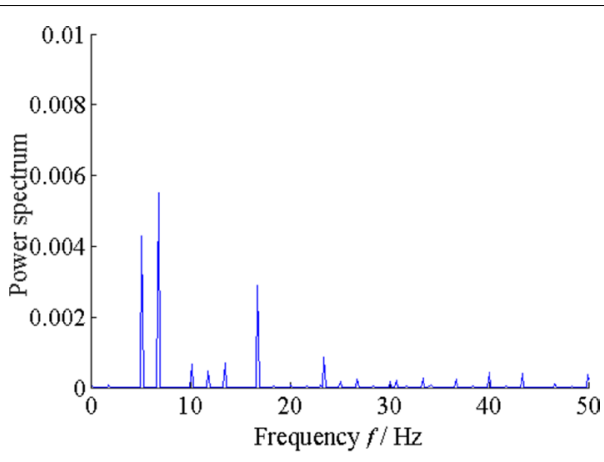

a Spectrum of the intact gear

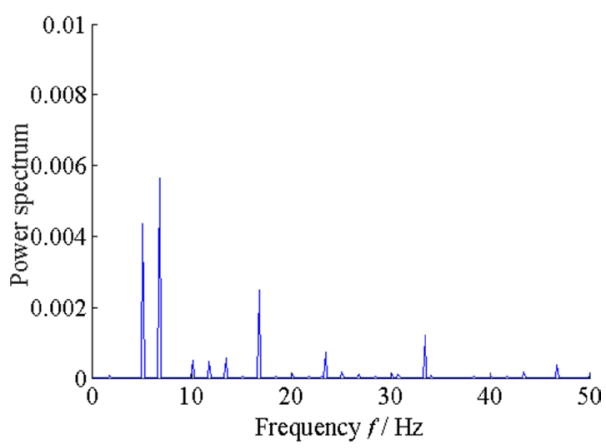

b Spectrum of the crack defect

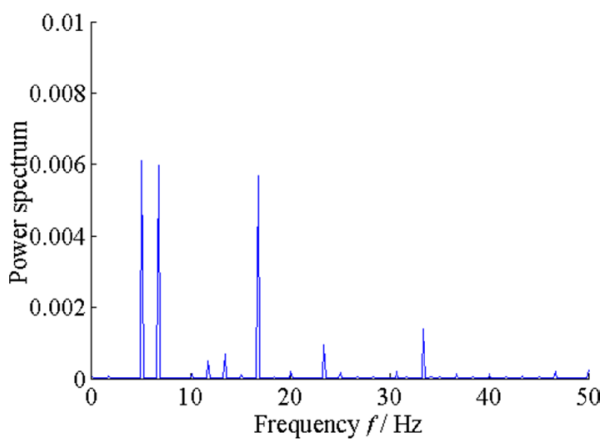

c Spectrum of the breakage defect

Figure 8 Spectra of differential signals at a speed of $17 \mathrm{~Hz}$

revolution are mounted on the input and output shafts, respectively. The signal of the encoder is collected by an NI 6211 DAQ with counter mode.

The intact gear, the cracked gear with a crack depth of $1 \mathrm{~mm}$ at the root of a single tooth, and the broken gear with one tooth completely removed are located on the input shaft from left to right (as shown in Figure 5). Meanwhile, the speeds of the input shaft are approximately 10 and $17 \mathrm{~Hz}$, respectively, and a load torque of $5 \mathrm{Nm}$ is applied to the output shaft. The signals of the

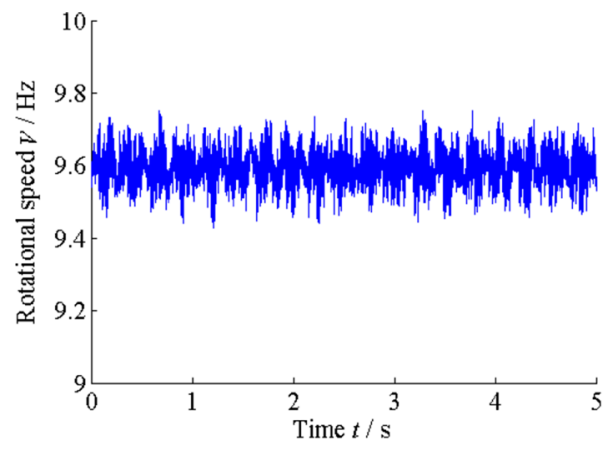

a Waveform of the intact gear

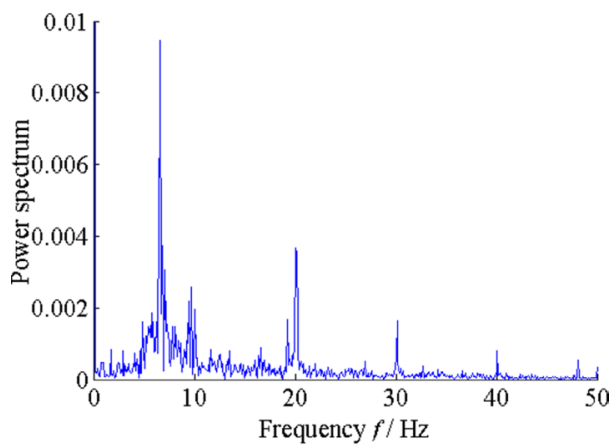

b Spectrum of the intact gear

Figure 9 Instantaneous speed signal of the input shaft encoder for the intact gear

two encoders are sampled by the $80 \mathrm{MHz}$ high-frequency counter, and the sample length is set to 102400 .

\subsection{Analyses and Discussions}

The waveforms and spectra of the instantaneous speeds collected from the three running conditions at an input speed of $10 \mathrm{~Hz}$ are shown in Figures 6 and 7, respectively. According to the gearbox structure and transmission ratio, the rotational frequency $f_{i}$ of the input shaft is $10 \mathrm{~Hz}$; the lay frequency $f_{m}$ and the output frequency $f_{o}$ are 4 and $3 \mathrm{~Hz}$, respectively.

The waveform of an intact gear, shown in Figure 6(a), is characterized by cyclical fluctuations; however, from the waveform of the crack defect given in Figure 6(b), some weak impulses can be observed in the speed fluctuation. On the contrary, as shown in Figure 6(c), owing to the reduced meshing stiffness, the distinct impulse caused by the broken tooth can be found.

Regardless of the gear state (intact gear, cracked gear, broken tooth), for the low frequency range of $[0,50]$ $\mathrm{Hz}$ in Figure 7, the main gear frequencies are denoted by arrows and are visible as being located at the primary shaft rotational frequency associated with 10 , 4, and $3 \mathrm{~Hz}$. Obviously, for the gear transmission, the 


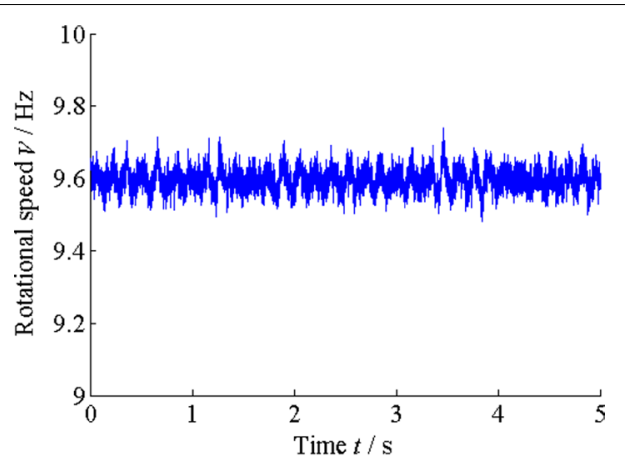

a Waveform of the crack defect

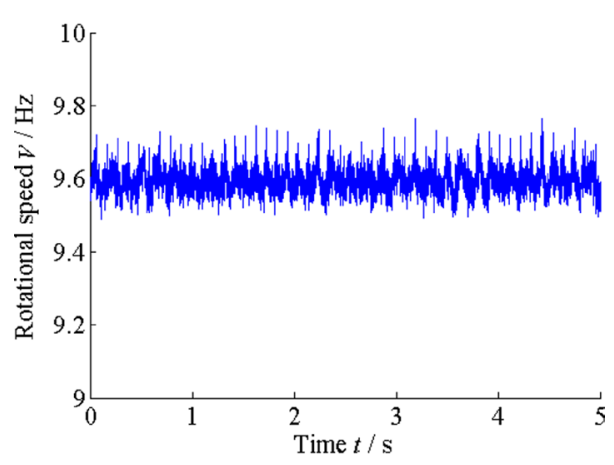

b Waveform of the breakage defect

Figure 10 Instantaneous speed signal of the input shaft encoder for localized defects

instantaneous speed contains all stage frequency components due to the excitation of manufacturing or installation errors, such as eccentricity of the wheel. However, there are no obvious harmonics of the rotary frequency in Figure 7(a) for the intact gear.

When a gear has a localized defect, the periodic excitation of the reduced mesh stiffness of the defect gear changes the performance of the rotational frequency and results in a harmonic component due to the time delay of the common-frequency excitations. As shown in Figure $7(\mathrm{~b})$, the amplitude of the input shaft frequency of $10 \mathrm{~Hz}$ is similar to that of the intact gear, but the second harmonic component can be observed in the spectrum of the crack defect. Meanwhile, for the breakage defect given in Figure 7(c), the amplitude of the input shaft frequency of $10 \mathrm{~Hz}$ is higher than that of the intact case because of the intense periodic impulses caused by broken teeth meshing. Thus, the fault of a broken tooth can be traced clearly by the highlighted frequency and its harmonic.

It can be concluded from the results that the differential signal of instantaneous speed can be used as an effective means to reflect the varying instantaneous speeds

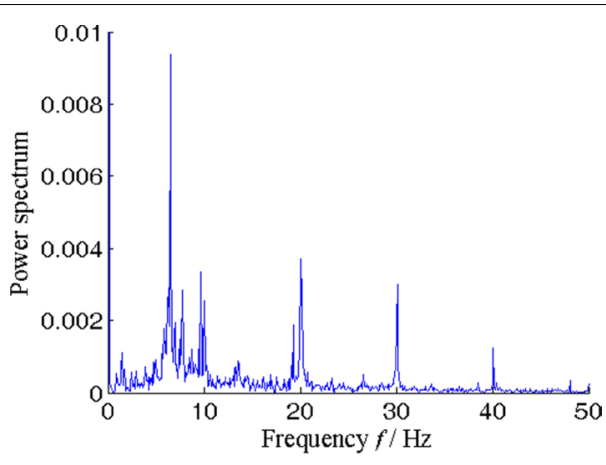

a Spectrum of the crack defect

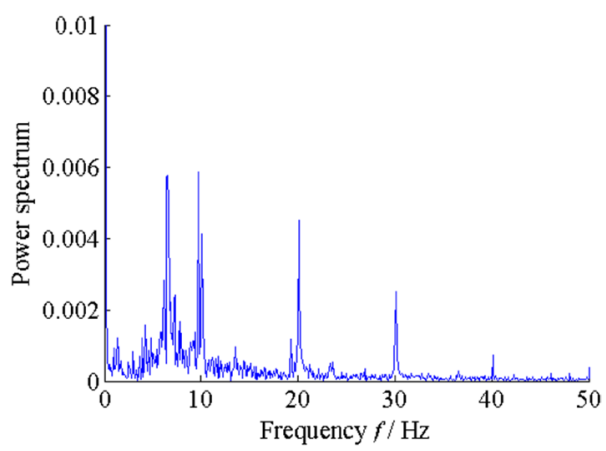

b Spectrum of the breakage defect

Figure 11 Spectra of instantaneous speed of the input shaft encoder

caused by localized defects, and to improve the detection ability for gear diagnosis.

Additionally, the instantaneous speed signals of double encoders at an input speed of $17 \mathrm{~Hz}$ are analyzed, and the spectra of the differential signals of the instantaneous speeds are given in Figure 8. For the input speed of $17 \mathrm{~Hz}$, three frequency components (input frequency $17 \mathrm{~Hz}$, lay frequency $6.8 \mathrm{~Hz}$, and output frequency $5.1 \mathrm{~Hz}$ ) are observed clearly.

It can be seen from Figure 8 that the spectrum of the intact gear only contains the frequencies of the input shaft, lay shaft, and output shaft, whereas the local defect excites the rotary frequency and its harmonic of the defect gear shaft.

\subsection{Comparison with Instantaneous Speed of Single Encoder}

To test the effectiveness of the proposed method, the instantaneous rotational speed of a single encoder is used. For the input speed of $10 \mathrm{~Hz}$, Figures 9, 10 and 11 depict the waveforms and spectra of the instantaneous speeds of the input shaft encoder in the intact and localized defect states. It is clear that the waveform of the 


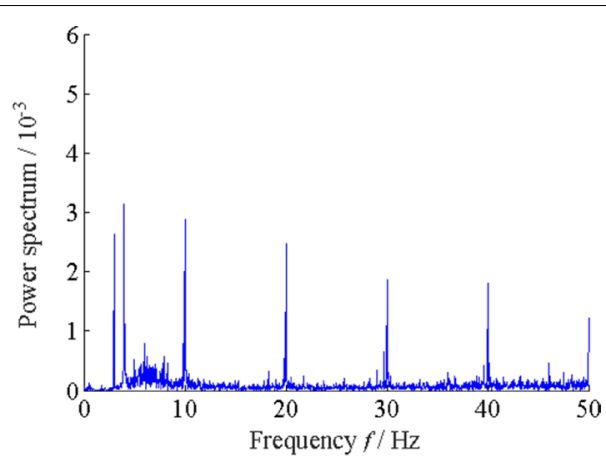

a Spectrum of the intact gear

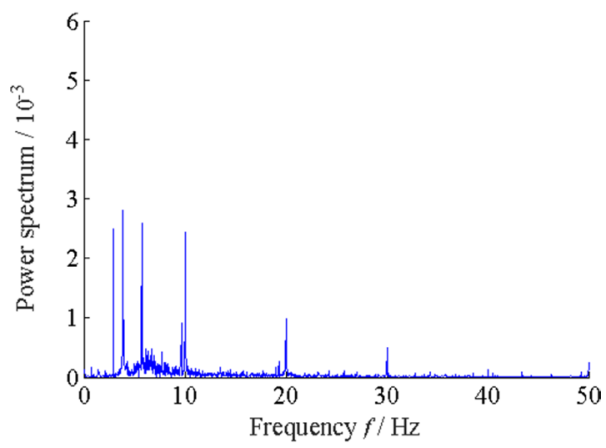

b Spectrum of the crack defect

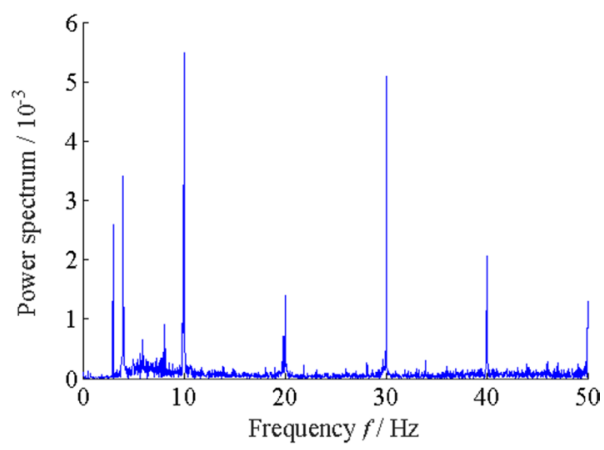

c Spectrum of the breakage defect

Figure 12 Spectra of instantaneous speed of the output shaft encoder

intact gear shown in Figure 9(a) is similar to that of the crack defect given in Figure 10(a). However, it is noted that the periodic impulses can be observed clearly for the broken tooth defect shown in Figure 10(b).

In the spectrum of the defect case shown in Figure 11, the frequency component of $10 \mathrm{~Hz}$ is the rotating frequency of the input shaft, whereas the predominant frequency of $6.5 \mathrm{~Hz}$ is the interference component inspired

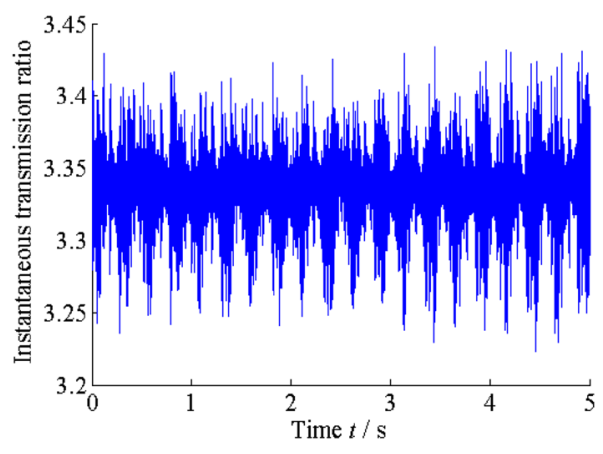

a Waveform of the intact gear

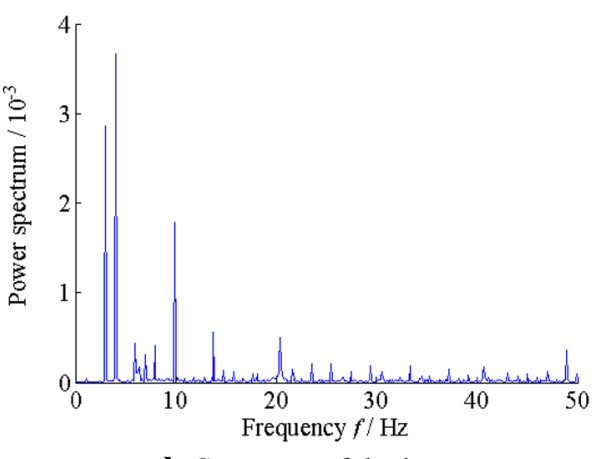

b Spectrum of the intact gear

Figure 13 Signal of instantaneous transmission ratio for the intact gear. a Waveform of the intact gear, $\mathbf{b}$ Spectrum of the intact gear

by other excitation in the gear chain, which covers the frequency components of the lay shaft and output shaft.

With increasing degree of gear defect, the amplitude of the frequency of $10 \mathrm{~Hz}$ increases gradually; This is consistent with the previous results. However, for the crack defect, it is difficult to use the instantaneous speed of the single encoder to identify faults as they have similar amplitude of rotational frequency.

Additionally, Figure 12 illustrates the spectra of instantaneous speed signals of the output shaft encoder in the intact and damaged conditions, respectively. In the case where the transmission ratio is considered, the varying speed of the output shaft is similar to that of the input shaft. It is noted that the excitation response of the gear chain appears in not only the speed signal of the input shaft, but also that of the output shaft. From the spectrum of the output shaft encoder, the corresponding frequency of $10 \mathrm{~Hz}$ has a significant increase for the broken tooth defect. 


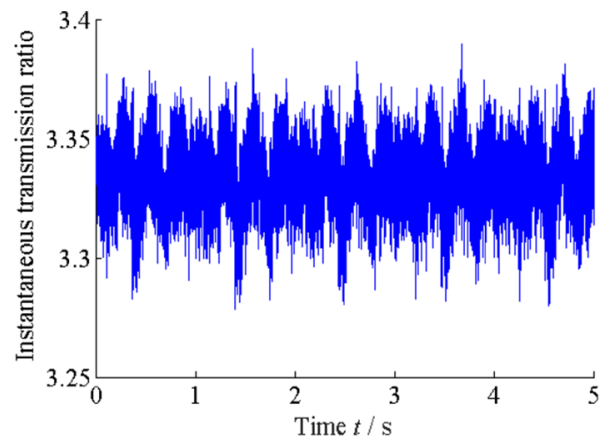

a Waveform of the crack defect

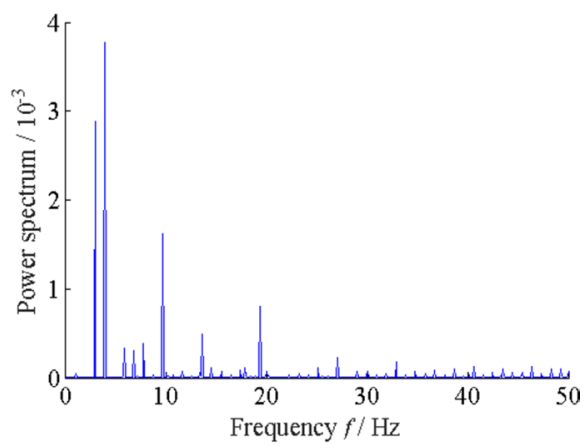

b Spectrum of the crack defect

Figure 14 Signal of instantaneous transmission ratio for the crack defect

However, for the crack defect, the amplitude of the $10 \mathrm{~Hz}$ frequency is similar to that of the intact gear, such that it is difficult to identify the fault from the signal of the intact gear. Obviously, compared to the single encoder, the differential speed signal of the instantaneous speeds can reduce the effect of external interference, and the crack defect can be identified by the exact frequency component.

\subsection{Comparison with Instantaneous Transmission Ratio}

The instantaneous transmission ratio is also used for comparison. For the sampling pairs of $V_{i}(n)$ and $V_{o}(n)$, the instantaneous transmission ratio can be defined as:

$$
I\left(n_{i}\right)=\frac{V_{i}\left(n_{i}\right)}{V_{o}\left(n_{o}\right)},
$$

where $V_{i}\left(n_{i}\right)$ is the $n$th instantaneous speed point of the input shaft, and $V_{o}\left(n_{o}\right)$ is the $n$th instantaneous speed

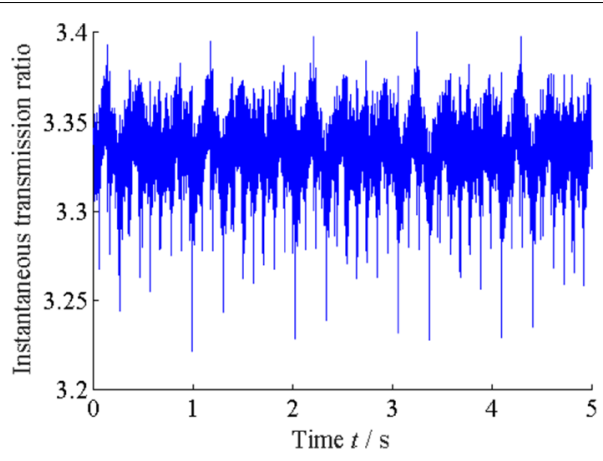

a Waveform of the breakage defect

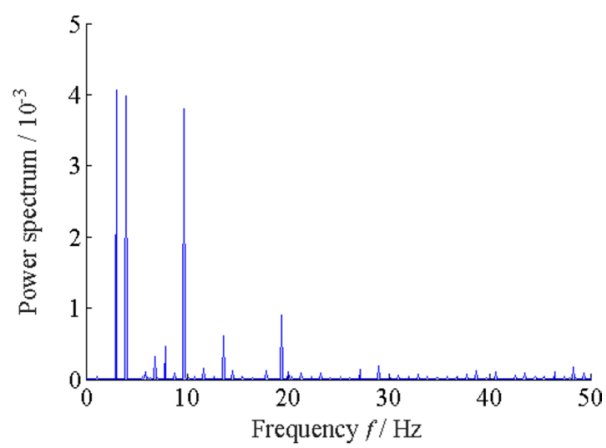

b Spectrum of the breakage defect

Figure 15 Signal of instantaneous transmission ratio for the breakage defect

point of the output shaft. The signal of the instantaneous transmission ratio for the intact gear and localized defects at the input speed of $10 \mathrm{~Hz}$ are shown in Figures 13, 14 and 15.

As shown in Figure 15, compared with the intact gear, the breakage defect is clearly observed in the form of the impulse, and the amplitude of the rotational frequency of $10 \mathrm{~Hz}$ also increases. However, for the crack defect, the rotational frequency and the second harmonic presented in Figure 14(b) are similar to that of the intact gear; thus, they are hardly distinguished. In brief, it is not easy to use the signal of the instantaneous transmission ratio to detect weak faults.

\subsection{Comparison with Vibration Signal}

For further comparison, the vibration in the test system is measured. The input speed is maintained at $17 \mathrm{~Hz}$ and a $5 \mathrm{Nm}$ load is imposed on the output shaft. Vibration 


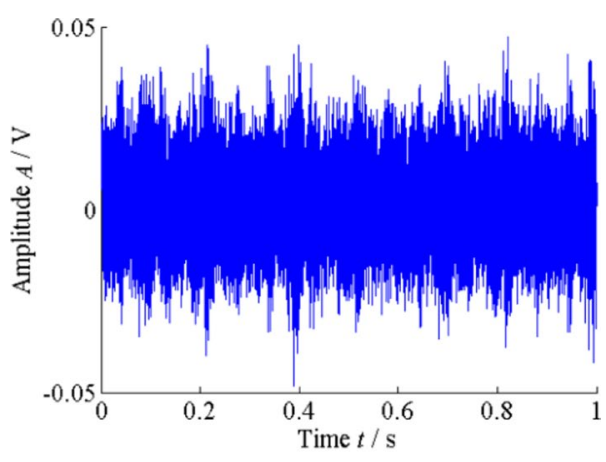

a Waveform of the intact gear

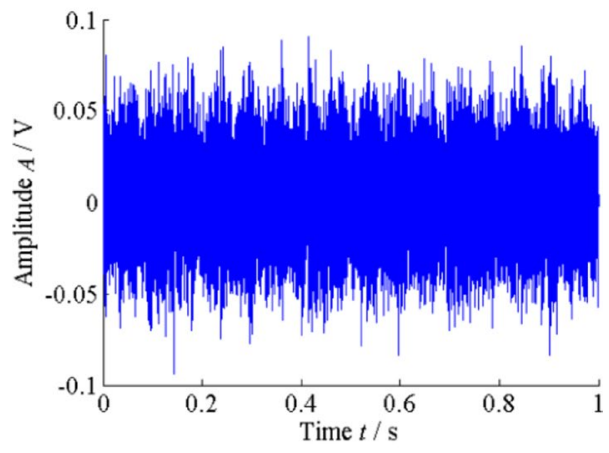

b Waveform of the crack defect

Figure 16 Waveform of the intact gear and the crack defect

signals are collected using an NI 9234 DAQ recorder at the sampling frequency of $10 \mathrm{kHz}$ for the input bearing. The waveforms of the intact gear and crack defect are given in Figure 16. It is obvious that the amplitude of the crack defect is still similar to that of the intact gear.

Considering the characteristic of the impulse caused by a localized fault in the gearbox or bearing [30], the Morlet wavelet is adopted to extract the fault feature from the vibration signal. Meanwhile, to extract the weak impulse component effectively, the shape factor of the Morlet wavelet can be optimized with the Shannon entropy.

With a shape factor of 0.4 , the optimal Morlet wavelet decomposition of the crack defect is shown in Figure 17. In the figure, the duration of the impulses corresponding to the rotation period of the driving shaft is not clear. Obviously, it is difficult to identify the crack defect with the time-frequency representation.

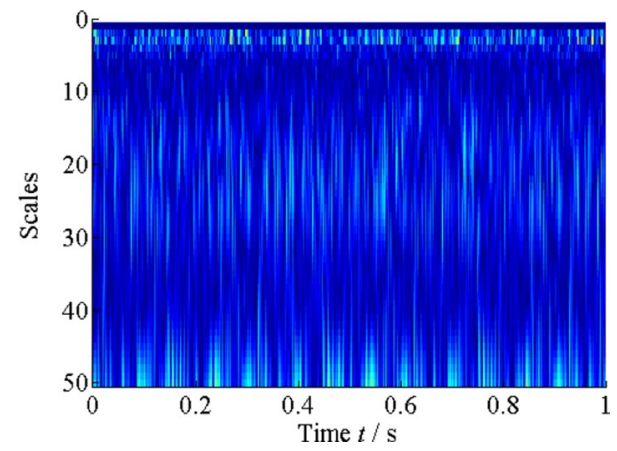

Figure 17 Time-frequency representation of the crack defect

\section{Conclusions}

(1) Based on the timer/counter-based method, the instantaneous rotational speeds of the input and output shafts can be obtained synchronously from the optical encoder. Meanwhile, the differential signal of instantaneous speed can reduce the effect of external excitations and enhance the extraction ability of the localized fault feature.

(2) For localized defects, the periodic excitation of the reduced mesh stiffness of the defect gear changes the spectrum performance of the rotational frequency and results in a harmonic component with the effect of same frequency excitations.

(3) Compared with the single encoder signal and the instantaneous transmission ratio, the differential signal of instantaneous speed is less affected by the external excitation. In addition, the optical encoders can provide a higher quality signal compared to vibration analysis.

\section{Authors' Contributions}

LL and GX was in charge of the whole trial; FL wrote the manuscript; XK and ML assisted with sampling and laboratory analyses. All authors read and approved the final manuscript.

\section{Author Details}

${ }^{1}$ School of Mechanical Engineering, Xi'an Jiaotong University, Xi'an 710049, China. ${ }^{2}$ Key Laboratory of Education Ministry for Modern Design and Rotor-bearing System, Xi'an Jiaotong University, Xi'an 710049, China. ${ }^{3}$ Engineering Workshop, Xi'an Jiaotong University, Xi'an 710049, China. ${ }^{4}$ State Key Laboratory for Manufacturing Systems Engineering, Xi'an Jiaotong University, Xi'an 710054, China.

\section{Authors' Information}

Lin Liang, born in 1973, is currently an associate professor at School of Mechanical Engineering, Xi'an Jiaotong University, and Key Laboratory of Education Ministry for Modern Design and Rotor-bearing System, Xi'an Jiaotong University, China. He received his PhD degree from Xi'an Jiaotong University, China, in 2007. His research interests include mechanical fault diagnosis, test and detection.

Fei Liu, born in 1979, is currently a research assistant at School of Mechanical Engineering, Xi'an Jiaotong University, China. He received his PhD degree from Xi'an Jiaotong University, China, in 2016. His research interests include 
precision measurement, and dynamic performance testing of mechanical system.

Xiangwei Kong, born in 1991, is currently a MS candidate at School of Mechanical Engineering, Xi'an Jiaotong University, China. His research interest includes mechanical fault diagnosis.

Maolin Li, born in 1978, is currently a lecturer at Engineering Workshop, Xi'an Jiaotong University, China. She received her PhD degree from Xi'an Jiaotong University, China, in 2014. Her research interests include mechanical fault diagnosis, test and detection.

Guanghua Xu, born in 1964, is currently a professor at School of Mechanical Engineering, Xi'an Jiaotong University, and State Key Laboratory for Manufacturing Systems Engineering, Xi'an Jiaotong University, China. He received his PhD degree from Xi'an Jiaotong University, China, in 1995. His research interests include mechanical fault diagnosis and brain-computer interface technology.

\section{Competing Interests}

The authors declare that they have no competing interests.

\section{Funding}

Supported by National Natural Science Foundation of China (Grant No. 51575438), China Postdoctoral Science Foundation (Grant Nos. 2017M623159, 2018T111046), Shaanxi Provincial Postdoctoral Science Foundation of China (Grant No. 2017BSHEDZZ68).

\section{Publisher's Note}

Springer Nature remains neutral with regard to jurisdictional claims in published maps and institutional affiliations.

Received: 2 April 2018 Accepted: 25 January 2019

Published online: 11 February 2019

\section{References}

[1] Y G Lei, J Lin, M J Zuo, et al. Condition monitoring and fault diagnosis of planetary gearboxes: A review. Measurement, 2014, 48(1): 292-305.

[2] L F Villa, A Reñones, J R Peran, et al. Statistical fault diagnosis based on vibration analysis for gear test-bench under non-stationary conditions of speed and load. Mechanical Systems and Signal Processing, 2012, 29(5): 436-446

[3] Y G Lei, N P Li, J Lin, et al. Two new features for condition monitoring and fault diagnosis of planetary gearboxes. Journal of Vibration and Control, 2015, 21(4): 755-764.

[4] H Sun, Y Zi, J Yuan, et al. Undecimated multiwavelet and Hilbert-Huang time-frequency analysis and its application in the incipient fault diagnosis of planetary gearboxes. Journal of Mechanical Engineering, 2013, 49(3): 56-62. (in Chinese)

[5] X F Liu, S R Qin, B Lin. Hybrid wavelet packet-teager energy operator analysis and its application for gearbox fault diagnosis. Chinese Journal of Mechanical Engineering, 2007, 20(6): 79-83.

[6] V Sugumaran, D Jain, M Amarnath, et al. Fault diagnosis of helical gearbox using decision tree through vibration signals. International Journal of Performability Engineering, 2013, 9(2): 221-233.

[7] $\mathrm{H} \mathrm{Li}$, J Zhao, W Song. Method of planetary gearbox feature extraction and condition recognition based on EMD and EDT. Journal of Vibration and Shock, 2016, 35(3): 48-54.

[8] D J Yu, Y Yang, J S Cheng. Fault diagnosis approach for gears based on EMD and SVM. Journal of Mechanical Engineering, 2005, 41 (1): 140-144. (in Chinese)

[9] Y G Lei, D Han, J Lin, et al. Planetary gearbox fault diagnosis using an adaptive stochastic resonance method. Mechanical Systems and Signal Processing, 2013, 38(1): 113-124.

[10] J Li, X Chen, Z He. Multi-stable stochastic resonance and its application research on mechanical fault diagnosis. Journal of Sound and Vibration, 2013, 332(22): 5999-6015.
[11] S Zhou, Z Ren, G Song, et al. Dynamic characteristics analysis of the coupled lateral-torsional vibration with spur gear system. International Journal of Rotating Machinery, 2015, 2015(2): 1-14.

[12] Z Feng, M J Zuo. Fault diagnosis of planetary gearboxes via torsional vibration signal analysis. Mechanical Systems and Signal Processing, 2013, 36(2): 401-421.

[13] Z P Feng, F L Chu. Torsional vibration signal analysis method for fault diagnosis of planetary gearboxes. Proceedings of the CSEE, 2013, 33(14): 101-106. (in Chinese)

[14] H Henao, S H Kia, G Capolino. Torsional-vibration assessment and gearfault diagnosis in railway traction system. IEEE Transactions on Industrial Electronics, 2011, 58(5): 1707-1717.

[15] D Remond, J Antoni, R B Randall. Instantaneous angular speed (IAS) processing and related angular applications. Mechanical Systems and Signal Processing, 2014, 45(1): 24-27.

[16] W G Yang, D X Jiang, T Han. Effects of tooth breakage size and rotational speed on the vibration response of a planetary gearbox. Applied Science, 2017, 7(7): 1-17.

[17] W Moustafa, O Cousinard, F Bolaers, et al. Low speed bearings fault detection and size estimation using instantaneous angular speed. Journal of Vibration and Control, 2016, 22(15): 3413-3425.

[18] A Fourati, N Feki, A Bourdon, et al. Electrical modeling for faults detection based on motor current signal analysis and angular approach. Proceedings of the Fourth International Conference on Condition Monitoring of Machinery in Non-Stationary Operations, Lyon, France, December 15-17, 2014: 15-25.

[19] Y Q Zhou, X S Mei, G D Jiang, et al. Technology on large scale numerical control machine tool condition monitoring based on built-in sensors. Journal of Mechanical Engineering, 2009, 45(4): 125-130. (in Chinese)

[20] M Zhao, J Lin, X F Wang, et al. Fluctuation analysis of instantaneous angular speed based on digital differentiator and its application in vibration source identification. Journal of Mechanical Engineering, 2012, 48(22): 1-6. (in Chinese)

[21] C J Stander, P S Heyns. Instantaneous angular speed monitoring of gearboxes under non-cyclic stationary load conditions. Mechanical Systems and Signal Processing, 2005, 19(4): 817-835.

[22] S K Roy, A R Mohanty, C S Kumar. Fault detection in a multistage gearbox by time synchronous averaging of the instantaneous angular speed. Journal of Vibration \& Control, 2016, 22(2): 1-13

[23] B Li, X N Zhang, J L Wu. New procedure for gear fault detection and diagnosis using instantaneous angular speed. Mechanical Systems and Signal Processing, 2017, 85(15): 415-428.

[24] K R Sankar, A R Mohanty, C S Kumar. Amplitude demodulation of instantaneous angular speed for fault detection in multistage gearbox. Proceedings of Vibration Engineering and Technology of Machinery, Manchester, UK, September 9-11, 2014: 951-961.

[25] A W Lees, M I Friswell, G Litak. Torsional vibration of machines with gear errors. Journal of Physics: Conference Series, 2012, 305(1): 789-798.

[26] F Chaari, W Baccar, M S Abbes, et al. Effect of spalling or tooth breakage on gear mesh stiffness and dynamic response of a one-stage spur gear transmission. European Journal of Mechanics, 2008, 27(4): 691-705.

[27] T Fakhfakh, F Chaari, M Haddar. Numerical and experimental analysis of a gear system with teeth defects. The International Journal of Advanced Manufacturing Technology, 2005, 5(5): 542-550.

[28] F S Gu, I Yesilyurt, Y H Li, et al. An investigation of the effects of measurement noise in the use of instantaneous angular speed for machine diagnosis. Mechanical Systems and Signal Processing, 2006, 20(6): 1444-1460.

[29] I Renaudin, F Bonnardot, O Musy, et al. Natural roller bearing fault detection by angular measurement of true instantaneous angular speed. Mechanical Systems and Signal Processing, 2010, 24(7): 1998-2011.

[30] H Qiu, J Lee, J Lin, et al. Wavelet filter-based weak signature detection method and its application on rolling element bearing prognostics. Journal of Sound and Vibration, 2006, 289(4): 1066-1090. 